\title{
Hybrid BSCF Genetic Algorithms in the Optimization of a PIFA Antenna
}

\author{
Mohammad Riyad Ameerudden and Harry C. S. Rughooputh
}

\begin{abstract}
With the exponential development of mobile communications and the miniaturization of radio frequency transceivers, the need for small and low profile antennas at mobile frequencies is constantly growing. Therefore, new antennas should be developed to provide both larger bandwidth and small dimensions.

This paper presents an intelligent optimization technique using a hybridized Genetic Algorithms (GA) coupled with the intelligence of the Binary String Fitness Characterization (BSFC) technique. The aim of this project is to design and optimize the bandwidth of a Planar Inverted-F Antenna (PIFA) in order to achieve a larger bandwidth in the $2 \mathrm{GHz}$ band. The optimization technique used is based on the Binary Coded GA (BCGA) and Real-Coded GA (RCGA). The optimization process has been enhanced by using a Clustering Algorithm to minimize the computational cost. During the optimization process, the different PIFA models are evaluated using the finite-difference time domain (FDTD) method.
\end{abstract}

Index Terms-BSFC, clustering, genetic algorithms, hybrid, intelligent computing.

\section{INTRODUCTION}

The Planar Inverted-F Antenna (PIFA) is the most widely used antenna owing to its low profile, simple structure and ease of fabrication, and primarily its high efficiency and wideband characteristic. Recently, PIFAs have been drawn much attention in antenna design and manufacturing as published in [1] and [2] papers. High gain of antennas, which is an important characteristic in terms of their performance, may only be attained through proper design and structure. However, there are many parameters, such as the sizes of the radiating elements, position of feeding wires, etc. that challenge engineers and manufacturers to design smaller antennas. The objective of this work is to maximize the bandwidth of a PIFA antenna while keeping its overall size small. While doing so, the optimization techniques have been analyzed to find a better convergence mechanism when applied to the modeling method.

\section{A. Modeling Method}

To analyze electromagnetic propagation in space, there exist different kind of three-dimensional full-wave methods

Manuscript received September 19, 2012; revised November 12, 2012. This work was supported in part by the Department of Electronics and Communications of the University of Mauritius and the Tertiary Education Commission (TEC) of Mauritius.

Mohammad Riyad Ameerudden is with the University of Mauritius, Mauritius (e-mail: riyadxxx@intent.mu).

Harry C. S. Rughooputh is with the Department of Electronics and Communications, University of Mauritius, Mauritius (e-mail: r.rughooputh@uom.ac.mu). and among these, three methods have become the most popular: the Finite Element Method (FEM), the Transmission Line Matrix (TLM) and the Finite Difference Time Domain (FDTD) method.

The application of these methods requires the use of powerful computers and delivers good approximation of electric and magnetic field propagation. To evaluate the performance of the antenna and observe the three-dimensional propagation of the electric and magnetic fields, the FDTD method was used.

\section{B. Optimization Techniques}

Owing to nonlinearities and complex interactions among problem variables, a search space may have more than one optimal solution. When solving problems, there is no escape if traditional methods get attracted to any of these locally optimal solutions.

Evolutionary algorithms, on the other hand, are based on the principles of biological evolution as explained [3]. Genetic algorithms (GA) are a class of evolutionary algorithm which provides optimization capabilities to a wide range of problems. Some of the issues that affect the traditional tools also affect GAs, but GAs have proved to be far more robust at handling complex and non-linear problems. The GA can providentially alleviate the difficulties of the sub-optimal solution. In this work, an intelligent GA technique has been experimented in an attempt to find out the best optimization mechanism for the problem.

\section{Methodology}

The method used in this project involves the modeling of the PIFA using the FDTD method through which the bandwidth of the antenna is evaluated. The bandwidth is adjusted by varying some of the key parameters of the antenna in the optimization process so as to converge to the optimal performance. As part of the optimization work, different GA techniques have been experimented to analyze the convergence behavior towards the best solution.

\section{A. Implementation of the FDTD}

FDTD starts by discretizing a three-dimensional space into rectangular cells, which are called Yee Lattice [4]. The Yee lattice is specially designed to solve vector electromagnetic field problems on a rectilinear grid. The grid is assumed to be uniformly spaced, with each cell having edge lengths $\Delta x, \Delta y$ and $\Delta z$. Fig. 1 shows the positions of fields within a Yee cell. 


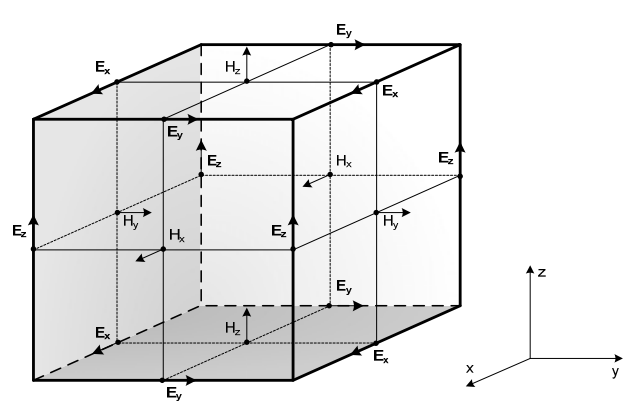

Fig. 1. An FDTD cell or Yee cell showing the positions of electric and magnetic field components.

Every $E$ component is surrounded by four circulating $\mathrm{H}$ components. Likewise, every $\mathrm{H}$ component is surrounded by four circulating $\mathrm{E}$ components. In this way, the curl operations in Maxwell's equations can be performed efficiently. Arrays must be used to represent the discrete space into a high-level programming language. One-dimensional space is represented by a 1D array, similarly 2D and 3D discrete spaces are represented by 2D and $3 \mathrm{D}$ arrays respectively. As explained in [5], the electric and magnetic equations are expressed in Array Space as

$$
\begin{aligned}
H_{x}^{n+\frac{1}{2}}(i, j, k)=H_{x}^{n-\frac{1}{2}}(i, j, k) & -\frac{\Delta t}{\mu}\left[\frac{E_{z}^{n}(i, j+1, k)-E_{z}^{n}(i, j, k)}{\Delta y}\right] \\
+ & \frac{\Delta t}{\mu}\left[\frac{E_{y}^{n}(i, j, k+1)-E_{y}^{n}(i, j, k)}{\Delta z}\right]
\end{aligned}
$$

$$
\alpha=\frac{\frac{\varepsilon^{\prime}}{\Delta t}-\frac{\sigma_{e q}}{2}}{\frac{\varepsilon^{\prime}}{\Delta t}+\frac{\sigma_{e q}}{2}} \quad \beta=\frac{1}{\frac{\varepsilon^{\prime}}{\Delta t}+\frac{\sigma_{e q}}{2}} \text {. }
$$

After having discretized the computational space and time, the FDTD has to be applied to the PIFA in order to simulate the propagating E-fields and $\mathrm{H}$-fields. The structure of the PIFA varies according to the different context in which it is used. This work deals only with the basic geometry of a PIFA which normally consists of a ground plate, a radiating plate and a feeding wire.

In order to excite the PIFA structure, ideally the field distribution of the dominant mode in the plane of excitation would be used. However, this distribution is not accurately specified for an arbitrary geometry. Instead, a y-directed electric field can be used to excite the antenna. A Gaussian pulse implemented as soft source is used as the excitation source.

The Voltage Standing Wave Ratio (VSWR) is the key to obtaining the bandwidth of the PIFA and thus, the key to achieve the objective of this project. In order to obtain the VSWR, the input impedance of the PIFA has first to be determined. The generalized input or line impedance can be simply calculated using the line voltage and current at a fixed point on the transmission line. These are obtained by Fourier transforming the time-dependent voltages and currents. Using the input impedance calculated, the S11 parameter can be obtained and consequently the VSWR is calculated as

$$
V S W R=\frac{1+\left|S_{11}\right|}{1-\left|S_{11}\right|}
$$

\section{B. Implementation of the GA}

The GA is the engine driving the optimization process and the FDTD modeling forms part of the fitness evaluation of the optimization. The GA begins its optimization with an initial random population, evaluates the fitness of each solution and selects the best ones for convergence towards the optimal solution, which will result to the best bandwidth performance, therefore the optimal antenna design. Following the previous work done in this area where different techniques were studied, this work presents further experimentation which involves the BSFC. In this work, the GA has been enhanced using a novel hybrid technique combining both BCGA and RCGA. As this is a binary problem, the BSFC, when using the proposed CPS method, consist of ones representing correct outputs and zeros representing outputs which are incorrect. For the PIFA consisting of 5 different parameters, the BSFC length was taken as 32 bits.

1) $B S F C$

In GA, selecting parents based on their fitness value, whether using absolute values, tournaments or a ranking system, is by far one of the most important conditions to satisfy in order to have a population evolving in the right direction. However, as demonstrated in [6] considering an individual in terms of a single value can be often limiting. In a typical GA problem, an individual may be very good at certain aspects of the problem and very poor at others. Consequently, considering only a consolidated overall fitness value for an individual may ignore the individual's detailed task-wise performance. In this regards, this technique has been applied in this project and the population strengths and weaknesses has been considered for the fitness evaluation.

As part of this process, an efficient pairwise parent selection process, the Comparative Partner Selection (CPS) [6] has been used for the crossover operation. This method aims to minimize the population variance throughout the iteration process. The objective is to maintain the search period of the optimization process, while individuals that do not satisfy all training cases equally (i.e., having a BSFC consisting of ones) do not dominate the population. Unlike other proposed methods to maintain diversity as evolution proceeds, the process experimented in this work is an effective mechanism for problem decomposition. The idea is to maintain a population that is capable of solving all training cases equally and has a good overall fitness value. The probability of crossover is devised in such a way that two similar individuals in terms of BSFC is less likely to happen than the crossover of two individuals with considerable difference in weaknesses.

\section{2) Hybrid BSFC with clustering}

The analysis and work done in the fitness evaluation area [6], [7] has highlighted the benefits of the BSFC. However, when applied to the current problem, it has been observed that the performance varies, and in most of the cases the process of converging to the optimal solution takes longer, as compared to the previous techniques experimented.

Therefore, in order to optimize the process and to bring more intelligence to the computation, the Clustering algorithm has been applied along with the BSFC concept. The clustering GA helps to reduce the cost of evaluation and accelerate the 
convergence [8]. Fig. 2 illustrates the conventional GA and the clustered GA.
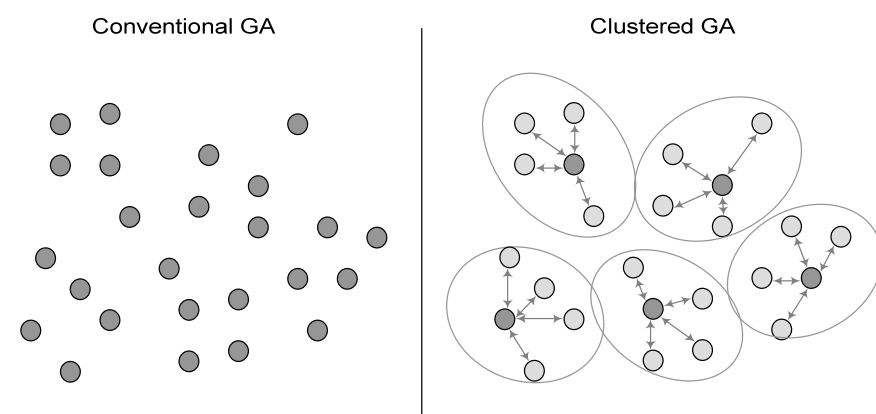

Fig. 2. Conventional GA vs. Clustered GA.

As illustrated in Fig. 3, clustering is a simple method of grouping the population into several small groups, called as clusters [9]. The algorithm evaluates only one representative for each cluster. The fitness of other individuals is estimated from the representatives' fitness. Using this method, large population can be maintained with reasonably less evaluation cost. One of the important factors to take into consideration for clustering is the similarity measure. This is commonly achieved using distance measures such as Euclidean distance, City block distance and Minkowski distance. Computation of the distance is generally done using equation

$$
d_{y}=d\left(X_{i}, X_{j}\right)=m \sqrt{\sum_{k=1}^{p}\left|x_{i k}-x_{j k}\right|^{m}}
$$

where $\mathrm{m}=1, \mathrm{~m}=2$ and $\mathrm{m} \geq 3$ for City block distance, Euclidean distance and Minkowski distance respectively. In order to adapt to the project situation, a combination of binary-coded and real-coded has been used. The BCGA has been used for the BSFC and evaluation process whereas the RCGA has been used for better clustering.

\section{EXPERIMENTAL RESULTS}

A succession of tests was carried out throughout the work to check whether the implementation of the FDTD was appropriate to evaluate the performance of the PIFA. These tests were carried out using different boundary conditions, different excitation pulses and different computational space size. The PIFA was excited using a Gaussian waveform of frequency ranging from $1.9 \mathrm{GHz}$ to $2.5 \mathrm{GHz}$. The feeding point, that is, the source location was varied by adjusting the parameters $\mathrm{fx}$ and $\mathrm{fz}$. The height of the radiating plate from the ground plate was also varied by changing the value of another parameter ' $h$ '. The variation of the height was quite small (approximately $2 \mathrm{~mm}$ ) since the idea of the project is to maximize the bandwidth of the PIFA while keeping the overall dimensions constant. Fig. 3 shows the electromagnetic propagation simulated from the FDTD. The bandwidth is defined by the range of frequencies where the VSWR is less than 2, which represents the $2 \mathrm{GHz}$ range.

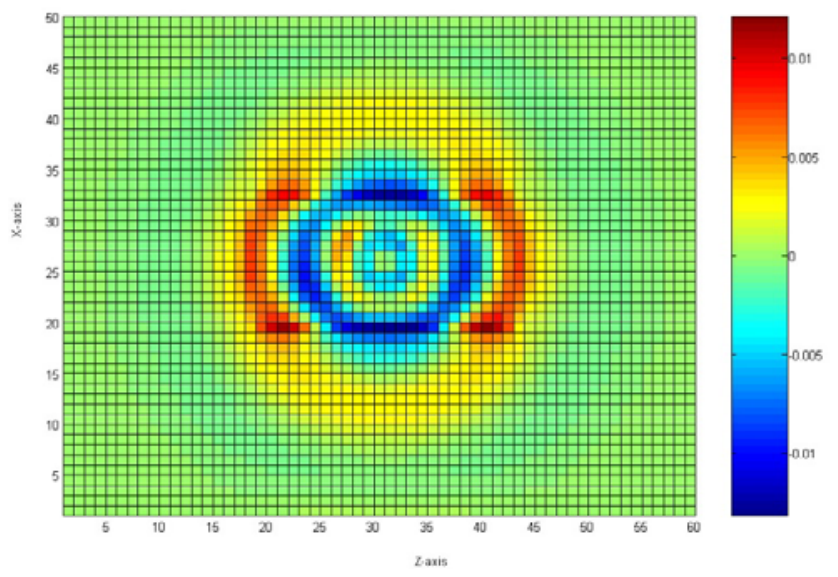

Fig. 3. Electromagnetic propagation from PIFA using FDTD.

A graph of VSWR against frequencies, as shown in Fig. 4, is plotted to show how the bandwidth is obtained.

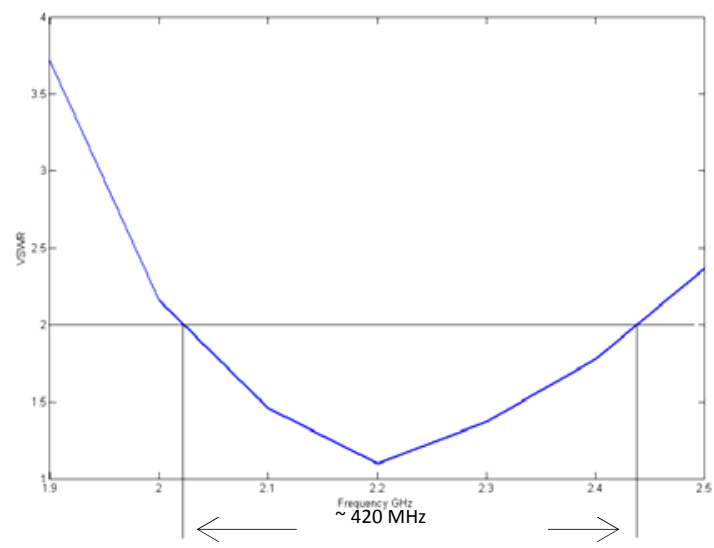

Fig. 4. Graph of VSWR v/s frequency.

The bandwidth obtained from the simulation is approximately $420 \mathrm{MHz}$. This is the optimal solution generated by the GA. The ground and radiating plates' dimensions were set to $50 \times 26 \mathrm{~mm}$ and $22 \times 14 \mathrm{~mm}$ respectively. The values of the parameters used for achieving this particular bandwidth are $\mathrm{fx}=3$ cells $(6 \mathrm{~mm}), \mathrm{fz}=3$ cells $(6 \mathrm{~mm})$ and $\mathrm{h}=4$ cells $(8 \mathrm{~mm})$. The results could be enhanced if the population size of the GA was bigger and if the number of discrete values used for the parameters were larger. However, as mentioned previously, this would cause the simulation to last much longer.

As for the GA process, the figures 5, 6 and 7 illustrate the results obtained from the different techniques which were experimented.

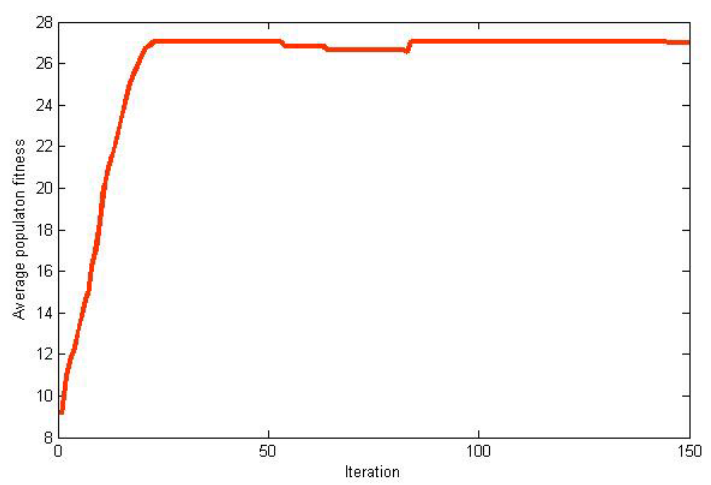

Fig. 5. Convergence without BSFC 


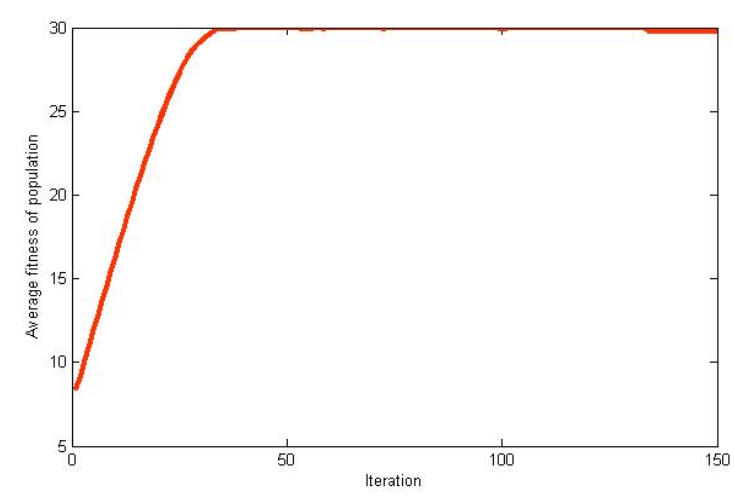

Fig. 6. Convergence with BSFC

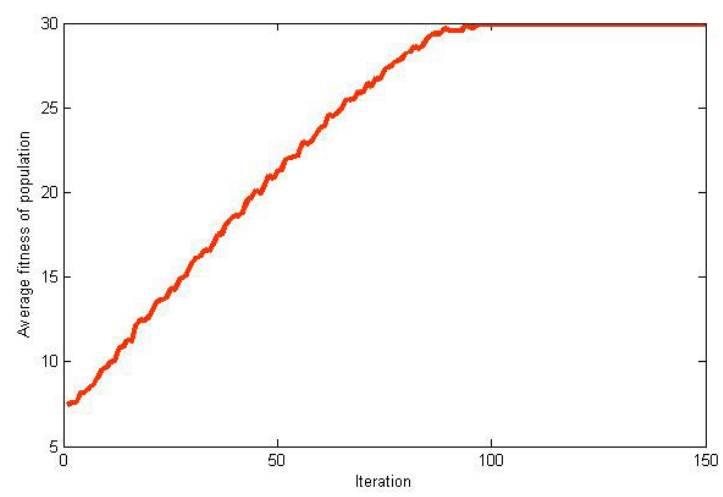

Fig. 7. Convergence with Hybrid BSFC

\section{CONCLUSION}

While the BCGA and RCGA have shown to be very good optimization methods, it has been observed that both may get stuck to sub optimal solution. The BSFC/CPS method was experimented with 150 runs for our PIFA problem and the convergence gain is visible in terms of average fitness and the number of runs ending in optimal (zero error) solutions is increased.

However, even though, the BSFC/CPS driven GA outperforms the others methods in terms of convergence, an increase in computational cost has been observed. In this regards, the clustering mechanism, has been applied. Consequently, this has led to an increase the number of iterations to reach the optimal solution but the computational cost is much lower. As such, a proper trade-off between convergence and performance could be observed.

\section{ACKNOWLEDGMENT}

M. R. A. Author thanks his wife, whose understanding and ability to put things back in perspective at critical times have been very helpful.

\section{REFERENCES}

[1] L. H. Wen, Y. Z. Yin, Y. Wang, J. X. Huang, X. S. Ren, and S. L. Zuo, "A novel PIFA antenna for broadband circular polarization," Microwave and Optical Technology Letters - Wiley Online Library, Nov 2010.

[2] Teruel O. Quevedo-, E. Pucci, and Iglesias E Rajo., "Compact Loaded PIFA for Multifrequency Applications," Antennas and Propagation, IEEE Transactions, vol. 58, no. 3, March 2010.

[3] D. Whitlet, K. Mathias., S. Rana, and J. Dzubera, "Evaluating evolutionary algorithms," Artifical Intelligence, 1996.

[4] K. S. Yee, "Numerical solution of initial boundary value problems involving Maxwell's equations in isotropic media," IEEE Trans. Antennas Propagat., vol. AP-14, pp. 302-307, May 1966.

[5] Gedney and Maloney, "Finite Difference Time Domain modeling and applications," FDTD Short Course, Mar. 1997.

[6] Peter Day and Asoke K. Nandi, "Binary String Fitness Characterization and Comparative Partner Selection in Genetic Programming," IEEE Trans. Evol. Comput, 2008. Jul. 10-12, 2000,

[7] R. I. (Bob) McKay, "Fitness sharing in genetic programming," in Proc. Genetic and Evol. Comput. Conf. (GECCO-2000), D. Whitley, D. Goldberg, E. Cantu-Paz, L. Spector, I. Parmee, and H.-G. Beyer, Eds., Las Vegas, NV, pp. 435-442.

[8] Seront, G. and Bersini, H., "A new GA-local search hybrid for continuous optimization based on multi level single linkage clustering," in Proc. GECCO-2000, pp .90 95, 2000.

[9] Gose, E., Johnsonbaugh, R. and Jost, S., "Pattern Recognition and Image Analysis," Prentice Hall PTR, 1996.

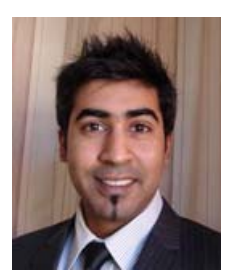

Mohammad R. Ameerudden was born in Mauritius on June 1981. This author has received his first class degree with honors in Bachelor of Engineering in 2004, from the Department of Electronics and Communications Engineering of the University of Mauritius. The author is currently undergoing post graduate $(\mathrm{PhD})$ research work in Genetic Algorithms and modeling techniques in the telecommunication and intelligent systems domain.

He has been in the information technology industry for $8+$ years and has experience in client interfacing, project management, feasibility studies, requirement elicitation and analysis, design and implementation of medium to large sized projects. As a Client Solution Manager at Infosys Ltd, Cyber city Mauritius, his current responsibility is for conceptualizing, articulating and delivering industry specific solutions, in Retail, CPG and Logistics industries. 